\title{
Long time diffusion in suspensions of interacting charged colloids
}

\author{
I M de Schepper†, E G D Cohen $\ddagger$, P N Pusey§ and \\ H N W Lekkerkerker|| \\ $\dagger$ IRI, University of Delft, 2629 JB Delft, The Netherlands \\ ¥ The Rockefeller University, 1230 York Avenue, New York, NY 10021, USA \\ $\S$ Royal Signals and Radar Establishment, Malvern, Worcestershire WR14 3PS, UK \\ \| Van't Hoff Laboratory, University of Utrecht, Padualaan 8, 3584 CH Utrecht, The \\ Netherlands
}

Received 23 June 1989

\begin{abstract}
A new expression is given for the long time diffusion coefficient $D_{\mathrm{L}}(k)$ of charged interacting colloidal spheres in suspension, as a function of the wavenumber $k$, near $k=k_{\mathrm{m}}$, where the static structure factor has a maximum. The expression is based on a physical analogy between a mode description of the behaviour of atomic fluids (as observed in neutron scattering) and of colloids (as observed in light scattering). Use of this expresssion in conjunction with a hard-sphere model yields good agreement with extant data on colloids.
\end{abstract}

The intermediate scattering function $F(k, t)$, the spatial Fourier transform of the densitydensity correlation function of monodisperse colloidal spheres in suspension as measured by light scattering, can be represented reasonably well by the sum of two simple exponential decays respectively defining a short time and a long time collective diffusion process [1-5]. The short time behaviour of $F(k, t)$ is for times $t_{\mathrm{B}}<t<t_{\mathrm{I}}$

$$
F(k, t)=S(k) \exp \left(-D_{\text {eff }}(k) k^{2} t\right)
$$

while for long times $t>t_{\mathrm{I}}$

$$
F(k, t) \sim \exp \left(-D_{\mathrm{L}}(k) k^{2} t\right)
$$

with $D_{\text {eff }}(k)$ and $D_{\mathrm{L}}(k)$ the short and long time diffusion coefficients and $S(k)=F(k, 0)$ the static structure factor of the particles, which characterises their spatial arrangement. The short time diffusion coefficient $D_{\text {eff }}(k)$ as a function of the wavenumber $k$ of the density (concentration) fluctuation is well understood. In the case, considered in this Letter, of dilute suspensions of charged particles that interact strongly through longrange repulsive electrostatic forces, but where hydrodynamic interactions may be neglected [5-7]

$$
D_{\text {eff }}(k)=D_{0} / S(k)
$$

where $D_{0}$ is the diffusion coefficient of a colloidal particle at infinite dilution due to Brownian motion. The time interval for which equation (1) is valid is large enough that Brownian motion (represented by $D_{0}$ in (3)) has established itself for a colloidal particle $\left(t>t_{\mathrm{B}}\right)$ in an external mean field exerted by the other particles (represented by $S(k)$ in 
(3)). However, the times $t$ in (1) are short enough that no significant displacements of the individual colloidal particles with respect to each other have occurred $\left(t<t_{\mathrm{I}}\right)$. Equation (3) has been well verified by light scattering experiments [2, 3, 8].

The long time behaviour of $F(k, t)$ has hitherto not been fully understood. Attempts by, among others, Felderhof and Jones [9] and Hess and Klein [10] have led to promising results, but no simple formula (like (3) for the short time behaviour) has emerged so far that would form a basis for a quantitative comparison with experiment.

Exploiting an analogy between the long time behaviours of $F(k, t)$ for dense atomic fluids and suspensions of interacting colloids, a new formula is obtained here for the long time diffusion coefficient $D_{\mathrm{L}}(k)$ in colloidal suspensions. This approach incorporates a simple physical description of the diffusion process and suggests moreover a relation between $D_{\text {eff }}(k)$ and $D_{\mathrm{L}}(k)$ that appears to agree with experiment. (The relation between this treatment and other analogies [5-7, 11] between the dynamics of atoms and colloids will be discussed elsewhere [12]). Both systems exhibit interacting diffusive behaviour over times spanning many collisions. The main difference is that, between collisions, atoms move ballistically whereas colloidal particles undergo Brownian diffusion.

We first discuss the atomic fluid. The long time behaviour of $F(k, t)$ in dense atomic fluids for $\sigma^{-1}<k<l_{\mathrm{E}}^{-1}$, which includes $k \cong k_{m} \cong 2 \pi / \sigma$ and for $t>t_{\mathrm{E}}$ is, in a hard sphere model of the fluid with particles of diameter $\sigma$, number density $n$, mean free path $l_{\mathrm{E}}$ and mean free time $t_{\mathrm{E}}$, given by [13-15]

$$
F(k, t) \sim \exp \left(-D_{\mathrm{L}}(k) k^{2} t\right)
$$

where the long time diffusion coefficient $D_{\mathrm{L}}(k)$ as a function of $k$ is approximately given by

$$
D_{\mathrm{L}}(k)=D(k) / S(k)
$$

with

$$
D(k)=D_{\mathrm{B}} d(k \sigma) / \chi
$$

Here $D_{\mathrm{B}}=0.216\left(k_{\mathrm{B}} T / m\right)^{1 / 2}\left(n \sigma^{2}\right)^{-1}$ is the Boltzmann self-diffusion coefficient (with $k_{\mathrm{B}}$ Boltzmann's constant, $T$ the temperature and $m$ the mass of the particles) and $\chi=g(\sigma)$ the radial distribution function $g(r)$ at contact $r=\sigma$. Physically, (6) decomposes the diffusion process in the fluid in two parts: $D_{\mathrm{E}}=D_{\mathrm{B}} / \chi$ is the high-density (Enskog) self-diffusion coefficient where $\chi$ increases the collision frequency in a dense fluid as compared to a dilute gas, and $d(k \sigma)$ incorporates the collisional transfer of momentum and energy at collision. Because of this transfer, the diffusion process is slowed down $(d(k \sigma)<1)$ when the wavelength $\lambda$ of a density fluctuation is near $2 \sigma(k \sigma \cong \pi)$ and enhanced $(d(k \sigma)>1)$ when $\lambda=\sigma(k \sigma=2 \pi)$. To a good degree of approximation $d(k \sigma)=\left(1-j_{0}(k \sigma)+2 j_{2}(k \sigma)\right)^{-1}$ where $j_{n}(k \sigma)$ is the spherical Bessel function of order $n$. $D(k)$ can be considered as a 'dressed' diffusion coefficient consisting of the 'bare' diffusion coefficient $D_{\mathrm{B}}$ multiplied by $d(k \sigma) / \chi$. It has been shown before that (4-6) describe the spectra observed in neutron scattering experiments in helium, neon, argon, krypton and rubidium for $k \cong k_{\mathrm{m}}$ very well [15].

In the analogy used here, the colloidal particles are replaced by hard spheres and the long time diffusion coefficient $D_{\mathrm{L}}(k)$ for the colloidal suspension is also given by (5) and (6) with $D_{\mathrm{B}}$ being replaced by $D_{0}$, the diffusion coefficient of a colloidal particle at infinite dilution,

$$
D_{\mathrm{L}}(k)=D_{0} d(k \sigma) / S(k) \chi .
$$

Comparing (3) and (7) we now see that the short and long time diffusion coefficients 

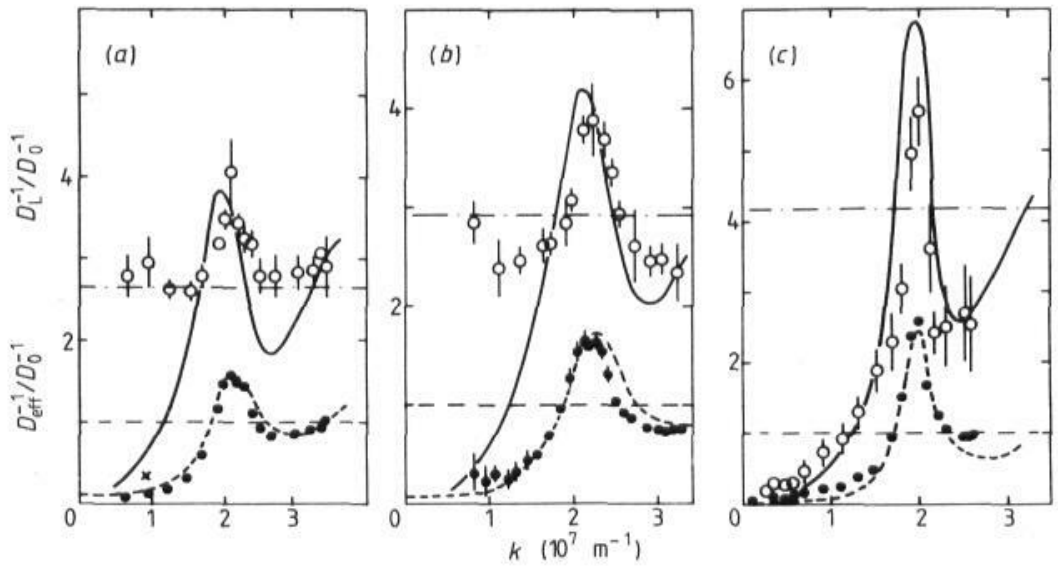

Figure 1. Reduced inverse diffusion coefficients $D_{\mathrm{L}}^{-1}(k) / D_{0}^{-1}$ (open circles) and $D_{\text {eff }}^{-1}(k) / D_{0}^{-1}$ (closed circles) as a function of $k$ for three solutions of polystyrene spheres in water ( $(a)$ Pusey [1]; (b) Dalberg et al [2]; (c) Grüner and Lehman [3]). The full curves are $D_{\mathrm{L}}^{-1}(k) / D_{0}^{-1}$ (cf equation (7)), the broken curves are $D_{\text {eff }}^{-1}(k) / D_{0}^{-1}=S(k)$ (cf equation (3)) and the chained horizontal lines are $D_{\mathrm{s}}^{-1} / D_{0}^{-1}=\chi$ (cf equation (9)) for equivalent hard sphere fluids with $\sigma=310 \mathrm{~nm}(a), 296 \mathrm{~nm}(b), 343 \mathrm{~nm}(c)$, and $n \sigma^{3}=0.59(a), 0.65(b) 0.82$ $(c)$. The horizontal lines at height 1 represent free Brownian motion. The data points for $D_{\mathrm{L}}^{-1}(k) / D_{0}^{-1}$ in $(a)$ and $(b)$ and $k<1.210^{7} \mathrm{~m}^{-1}$ have not been corrected for incoherent scattering, except for one point [5] (cross in $(a))$.

differ by the factor $d(k \sigma) / \chi$, which, qualitatively, can be said to describe the structural relaxation of a particle in its 'cage' of near neighbours.

Figure 1 shows three sets of experimental data for short and long time diffusion coefficients that were analysed in terms of the analogy developed above. The required hard-sphere diameters $\sigma$ and number densities $n$ were determined (as in [15] for atomic fluids) by fitting the experimentally observed $S(k)$ to a hard sphere $S(k)$. Since for hard spheres $S(k)$ is a function of $k \sigma$ and $n \sigma^{3}$ only, $\sigma$ and $n$ are obtained from these best fits. The resulting values of $n$ agree with independent experimental estimates and $\chi=\chi\left(n \sigma^{3}\right)$ is obtained from the hard-sphere equation of state.

The fitted hard-sphere structure factors are shown by broken lines in figure 1 . The solid lines show values of $D_{0} / D_{\mathrm{L}}(k)$ predicted by (7). Overall, agreement between experiment and theory is surprisingly good. The deviations in figures $1(a)$ and $(b)$ between the theoretical and experimental values of $D_{\mathrm{L}}(k)$ seen at small $k$ are most likely due to the appreciable polydispersity [5] of the colloidal particles used in the experiments. For, when $S(k)$ becomes very small $\left(k \ll k_{\mathrm{m}}\right)$ the experimentally measured $F(k, t)$ is increasingly dominated by the slowly decaying (incoherent) self-intermediate scattering function $F_{\mathrm{s}}(k, t)$ given by $(8)$ and $(9)$ below. The reason that such effects did not show up in the experiments of figure $1(c)$ is that a less polydisperse sample was used; note, however, that large corrections for multiple scattering were necessary in this experiment. To understand the long time diffusion coefficient $D_{\mathrm{L}}(k)$ given by $(7)$ for large $k$, one notices that the diffusion process will approach a self-diffusion process, where $S(k)=$ $d(k \sigma)=1$ and $F(k, t)$ approaches $F_{\mathrm{s}}(k, t)$ given for long times $t>t_{\mathrm{l}}$, by

$$
F_{\mathrm{s}}(k, t) \sim \exp \left(-D_{\mathrm{s}} k^{2} t\right) .
$$

Here $D_{\mathrm{s}}$ is the self-diffusion coefficient of a tagged colloidal particle, i.e.

$$
D_{\mathrm{s}}=D_{0} / \chi \text {. }
$$

Thus, for increasing $k>k_{\mathrm{m}}, D_{\mathrm{L}}(k)$ will approach $D_{\mathrm{s}}$, i.e., the full curves $D_{\mathrm{L}}^{-1}(k) / D_{0}^{-1}$ 
will approach the chained curves $D_{\mathrm{s}}^{-1} / D_{0}^{-1}=\chi$ in figure 1 . This approach is oscillatory, due to the oscillations in $S(k)$ and $d(k \sigma)$ [13]. A similar oscillating behaviour for $k>k_{\mathrm{m}}$ of $F(k, t)$ around $F_{\mathrm{s}}(k, t)$ has been observed by Sköld et al in liquid Ar, many years ago [16].

We further remark that the long time diffusion process at intermediate $k \sim k_{\mathrm{m}}$ is due to a (slow) structural relaxation, both in atomic fluids and in charged colloids. For atomic fluids, this has been discussed extensively before by two of us [13,15], while for colloids it has been mentioned by Hess and Klein [10]. In spite of the different language used in these two discussions the basic phenomenon is the same: while we use an extended heat mode, that behaves like a self-diffusion mode, Hess and Klein use a two viscoelastic mode description. As Kirkpatrick has shown [14] the two descriptions are equivalent when $k \sim k_{\mathrm{m}}$. Of course, away from $k \sim k_{\mathrm{m}}$ the two approaches lead to entirely different results. Finally, we note that the semi-quantitative analogy between charged colloids and atomic fluids via a hard-sphere model fluid is remarkable, in view of the absence of any adjustable parameters other than (realistic) hard-sphere diameters to characterise the interaction size of the particles and in view of the huge scaling factors between the two systems, namely, a diameter ratio of about 800 and an inverse self-diffusion coefficient ratio of about 400 leading to time scale ratios of the order of $10^{9}$. This will be discussed more extensively elsewhere [12].

One of us (EGDC) gratefully acknowledges the hospitality and the support of the Institute for Theoretical Physics and the University of Utrecht, where this work was initiated, as well as support from the US Department of Energy, under DOE grant no. DE-FG02-88ER13847. PNP and HNWL gratefully acknowledge financial support of NATO, through research grant 132/84.

\section{References}

[1] Pusey P N 1978 J. Phys. A: Math. Gen. 11119

[2] Dalberg P S, Boe A, Strand K A and Sikkeland T 1978 J. Chem. Phys. 695473

[3] Grüner F and Lehman W P 1982 J. Phys. A: Math. Gen. 152847

[4] Taylor T W and Ackerson B J 1985 J. Chem. Phys. 832441

[5] Pusey P N and Tough R J A 1985 Dynamic Light Scattering, ed. R Pecora (New York: Plenum)

[6] Ackerson B J, Pusey P N and Tough R J A 1982 J. Chem. Phys. 761279

[7] Tough R J A, Pusey P N, Lekkerkerker H N W and van den Broeck C 1986 Mol. Phys. 59595

[8] Brown J C, Pusey P N, Goodwin J W and Ottewill R H 1975 J. Phys. A: Math. Gen. 8664

[9] Felderhof B U and Jones R B 1983 Physica A 121 329; 12289

[10] Hess W and Klein R 1981 Physica A 105 552; 1983 Adv. Phys. 32173

[11] Cohen E G D, de Schepper I M and Campa A 1987 Physica A 147142

[12] Pusey P N, Lekkerkerker H N W, Cohen E G D and de Schepper I M 1989 unpublished

[13] de Schepper I M, Cohen E G D and Zuilhof M J 1984 Phys. Lett. A 101 399; Physica B 127282

[14] Kirkpatrick T R 1985 Phys. Rev. A 323130

[15] Cohen E G D, Westerhuijs P and de Schepper I M 1987 Phys. Rev. Lett. 592872

[16] Sköld K, Rowe J M, Ostrowski G and Randolph P D 1972 Phys. Rev. A 61107 